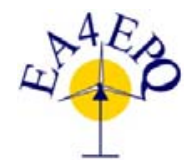

International Conference on Renewable Energies and Power Quality (ICREPQ'16)

Madrid (Spain), $4^{\text {th }}$ to $6^{\text {th }}$ May, 2016

Renewable Energy and Power Quality. Tournal (RE\&PQJ)

ISSN 2172-038 X, No.14 May 2016

\title{
Short-term load forecasting using an Artificial Neural Network for Battery Energy Storage System
}

\author{
Hyang-A Park ${ }^{1}$, Suel-Ki Kim ${ }^{1}$, Jong-yul Kim ${ }^{1}$, Jin-Wook Kim ${ }^{1}$, Kyeng-Hee Cho ${ }^{1}$ and Eung-Sang Kim ${ }^{1}$ \\ ${ }^{1}$ Smart Distribution Research Center \\ Koera Electrotechnology Research Institute \\ 12, Bulmosan-ro 10beon-gil, Seongsan-gu, Changwon-si, Gyeongsangnam-do(Korea) \\ Phone/Fax number:+82 055280 2438/+82 055280 1339, \\ e-mail: giddk100414@keri.re.kr, blksheep@keri.re.kr, ivkim@keri.re.kr,

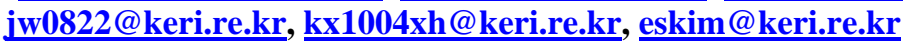

\begin{abstract}
Load levelling that use recent spotlighted the Battery Energy Storage System (BESS) saves spare electricity or low cost electricity. The saved electricity can be used for peak power demand time. In this paper, load is predicted for purpose of operating the BESS effectively. By analysing pattern of industrial load, it is classified into workdays, Saturday and holidays. Then apply ANN on workdays load pattern from classified load patterns to predict load. To increase accuracy, number of neurons in hidden layer and learning data period are changed for estimation of load. After that, error rate was calculated for comparison and analysis.
\end{abstract}

\section{Key words}

Load forecasting, Artificial Neural Network, Smart grid, Back propagation, Multi layer perceptron.

\section{Introduction}

Due to recent demand of electricity, importance of load estimation for application and securing consumption resources through reduction of electricity energy internationally. In technological perspective, it increases reliability of electricity supply and needs for safe operation of power generation system, prevention of emergency feeding status, estimation of peak and estimation of load curve. In economical perspective, it decreases cost of power generation and predict peak demand supply to save massive amount of burden every year. Like above, accuracy of load estimation is necessary for establishing rational power supply plan.

Accurate load estimation is necessary to construct rational power supply plan and operate a Demand Manageable Battery Energy Storage System that saves spare and low cost electricity, and use the saved electricity on peak demand time. To predict load which is Time series data, Exponential smoothing method, Autoregressive moving average model and State-space model are commonly used. To overcome basic limitation of time series prediction method, recently forecasting model with artificial intelligence method such as Artificial Neural Network (ANN)[1] and fuzzy theory are actively researched.

This paper classified load pattern into workdays, Saturday and holidays by analysing load pattern from load data of industrial load. Then applied to workdays load pattern. By using neural network, it was possible to predict load for charging and discharging of BESS and mean absolute percentage errors (MAPE) of predicted load is calculated and analyzed.

\section{Artificial Neural Network}

ANN is developed to imitate biological neural network. It is a model that make possible to do complex non-linear calculation with inspiration from biological structure and functions. Important feature of ANN, training is very useful to analyze non-linear pattern. During learning process of ANN, it renews connection weight to minimize errors between calculated output (predicted load value) by input and objective value (actual load value). For case of ANN, pre-information of domain is not necessary and it has advantage of building a prediction model with only input-output learning data, so it has been used for building a prediction model.

As methods of training ANN, there are perceptron learning rule, adaline (Adaptive linear element), WidrowHoff learning rule, Delta learning rule and back propagation. Its structure also various. This paper predicted load by using error back propagation algorithm from multi layer perceptron (MLP)[2] which is the most effective and common for prediction. Structure of multi layer perceptron (MLP) consists more than one hidden layer between input layer and output layer. Structure of common feedforward structure three-layer MLP is like Fig. $1[3],[4]$. 


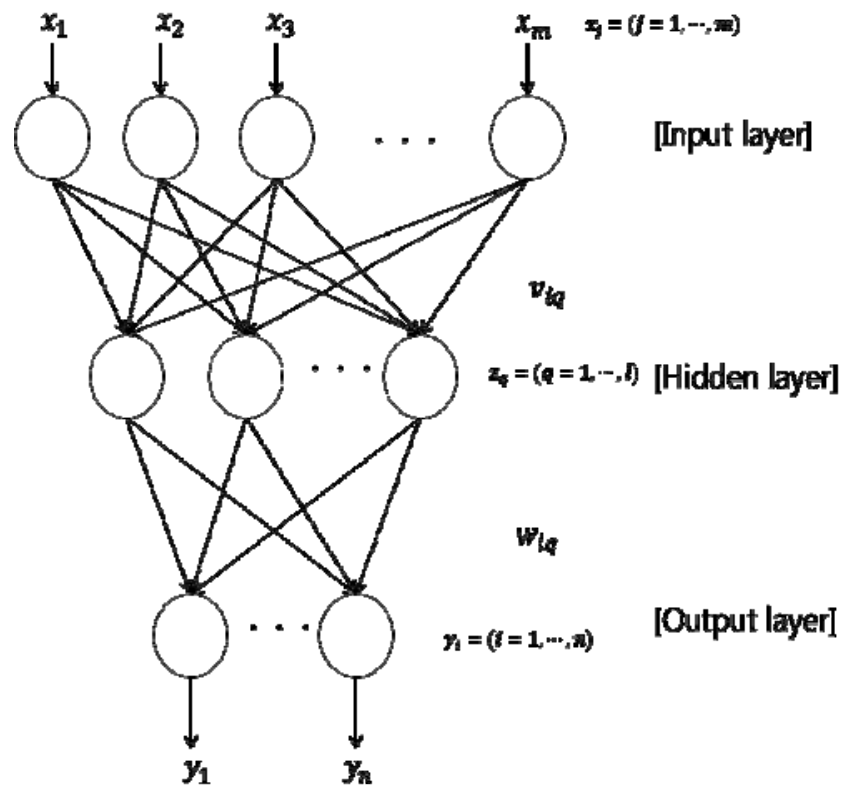

Fig. 1. Feed-forward artificial neural network structure

An output value of neuron in hidden layer is calculated like Equation (1), an output value of neuron in output layer is calculated like Equation (2).

$$
\begin{aligned}
& \mathrm{z}_{q}=a\left(\text { net }_{q}\right), \quad \text { net } t_{q}=\sum_{j=1}^{m} v_{q j} x_{j} \\
& y_{i}=a\left(\text { net }_{i}\right), \quad n e t_{i}=\sum_{q=1}^{l} w_{i q} z_{q}
\end{aligned}
$$

An objective function that minimize difference between output value of output layer and objective value is defined as Equation (3).

$$
\mathrm{E}=\frac{1}{2} \sum_{i=1}^{n}\left(d_{i}-y_{i}\right)^{2}
$$

A connection weight between input layer and hidden layer is defined as Equation (4).

$$
\begin{aligned}
\Delta w_{i q} & =-\eta \frac{\partial E}{\partial w_{i q}} \\
& =\eta\left\{\left(\mathrm{d}_{i}-y_{i}\right) \mathrm{a}^{\prime}\left(\text { net }_{i}\right)\right\} \mathrm{z}_{q}
\end{aligned}
$$

A connection weight between hidden layer and output layer is defined as Equation (5).

$$
\begin{aligned}
\Delta v_{q j} & =-\eta \frac{\partial E}{\partial v_{q j}} \\
& =-\eta x_{j} \mathrm{a}^{\prime}\left(\text { net }_{i}\right)\left(-\sum_{i=1}^{n} \delta_{o i} w_{i q}\right)
\end{aligned}
$$

$\delta_{o i}=\left(d_{i}-y_{i}\right) a^{\prime}\left(\right.$ net $\left._{i}\right)$

Where $x_{j}$ is input-variable, $z_{q}$ is Neuron's output of hidden later, $y_{i}$ is Model output, $v_{q j}$ is weight of input layer to hidden layer, $w_{i q}$ is weight of hidden layer to output layer, $d_{i}$ is desired output and $E$ is cost function. It is need to optimize an objective function, Equation (3), that update each connection weight to minimize error between output value and objective value[5].

\section{Case Study}

In this paper, load is predicted to apply predicted load from ANN to optimized charging and discharging operation plan of BESS. For that, load patterns on October 2014 are analyzed and classified as workdays, Saturday and holidays. Then applied to workdays load pattern from classified load pattern.

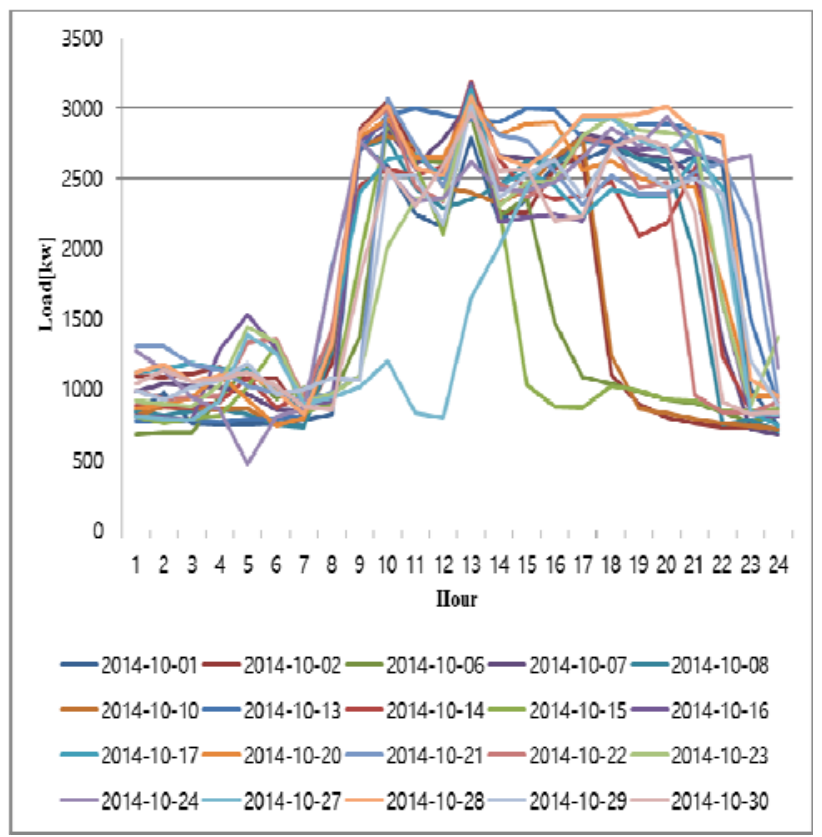

Fig. 2. Load pattern of Workdays(October, 2014)

Similar to Fig. 2, even workingdays load pattern is already classified load pattern, it is possible to confirm that each load is in very different shape. ANN with feedforward MLP structure is applied to workingdays load pattern on October 2014 to predict load. For an activation function of neuron in hidden layer and neuron in output layer, tangent sigmoid function and linear function are used respectively. For accuracy increase of ANN prediction, number of neuron in hidden layer is changed to predict load and compose many cases of learning time to predict load. Prediction error rate is calculated to confirm prediction accuracy of load that predicted from predicted load by ANN and moving average method. Then error rate is analyzed. Prediction error rate is defined as Equation (7) [6]. 


$$
\operatorname{MAPE}(\%)=\frac{\left|L_{\text {actual }}-L_{\text {forecastk }}\right|}{L_{\text {forecastk }}} \times 100
$$

A. Load forecasting for the number of neuron of hidden layers and the number of input data

Prediction accuracy of neural network can be different with number of neuron in hidden layer, so number of neuron in hidden layer is change to 1,2 and 3 to execute simulation. Number of input data is set as 3 (24 hours before load, 48 hours before load, 72 hours before load). Output function due to input data can be displayed as next.

$$
\hat{x}(t)=f(x(t-24), x(t-48), x(t-72))
$$

Load forecasting using Moving average method[7] is calculated like Equation (9).

$$
M A L_{k}=\frac{1}{n} \sum_{i=1}^{n} x_{i, k}
$$

Where $\mathrm{n}$ is period, $\mathrm{k}=1, \ldots, 24$ hour and $x_{i, k}$ is historical load data.

Table I. - MAPE of Load forecasting for Case 1, Case 2, Case 3 and Case 4

\begin{tabular}{|c|c|c|c|c|}
\hline & $\begin{array}{c}\text { MAPE of } \\
\text { Case 1 } \\
{[\%]}\end{array}$ & $\begin{array}{c}\text { MAPE of } \\
\text { Case 2 } \\
{[\%]}\end{array}$ & $\begin{array}{c}\text { MAPE of } \\
\text { Case 3 } \\
{[\%]}\end{array}$ & $\begin{array}{c}\text { MAPE of } \\
\text { Case4 } \\
{[\%]}\end{array}$ \\
\hline $2014-10-01$ & 16.8 & 16.2 & 15.6 & 16.2 \\
\hline $2014-10-02$ & 37.7 & 37.0 & 37.0 & 42.5 \\
\hline $2014-10-06$ & 46.1 & 45.7 & 46.1 & 43.7 \\
\hline $2014-10-07$ & 11.0 & 10.6 & 10.9 & 16.4 \\
\hline $2014-10-08$ & 13.1 & 12.6 & 12.0 & 18.9 \\
\hline $2014-10-10$ & 36.0 & 35.3 & 34.8 & 28.3 \\
\hline $2014-10-13$ & 19.9 & 19.5 & 19.3 & 18.7 \\
\hline $2014-10-14$ & 11.6 & 11.3 & 11.7 & 11.2 \\
\hline $2014-10-15$ & 57.0 & 56.5 & 56.0 & 56.4 \\
\hline $2014-10-16$ & 17.0 & 16.5 & 16.6 & 14.7 \\
\hline $2014-10-17$ & 15.1 & 14.3 & 14.7 & 15.5 \\
\hline $2014-10-20$ & 11.9 & 11.5 & 11.8 & 16.2 \\
\hline $2014-10-21$ & 21.0 & 10.5 & 21.2 & 17.8 \\
\hline $2014-10-22$ & 10.5 & 10.0 & 9.9 & 27.0 \\
\hline $2014-10-23$ & 20.4 & 19.8 & 19.9 & 20.7 \\
\hline $2014-10-24$ & 21.2 & 20.7 & 20.8 & 22.4 \\
\hline $2014-10-27$ & 46.3 & 45.7 & 44.9 & 43.8 \\
\hline $2014-10-28$ & 13.9 & 13.4 & 13.8 & 15.6 \\
\hline $2014-10-29$ & 17.1 & 11.5 & 11.6 & 18.7 \\
\hline $2014-10-30$ & 12.0 & 11.5 & 11.6 & 18.7 \\
\hline Total & 22.8 & 22.2 & 22.3 & 24.0 \\
\hline & & & & \\
\hline
\end{tabular}

1) Case 1: number of neuron in hidden layer is 1, number of input data is 3 and learning period is 30 days.
2) Case 2: number of neuron in hidden layer is 2, number of input data is 3 and learning period is 30 days.

3) Case 3: number of neuron in hidden layer is 1, number of input data is 3 and learning period is 30 days.

4) Case 4: load forecasting using Moving average method. Number of input data is 3 and period is 3 days.

Through Table I, it is possible to confirm that prediction error rate of predicted load with 3 input data and 2 neurons in hidden layer has the least value. For the case 2, MAPE's prediction error rate is $22.2 \%$, so it is possible to confirm that MAPE with Moving average method has $1.8 \%$ more value than predicted value. Also it is possible to confirm that prediction rate is the highest compare with other cases. Fig. 3. show load forecasting chart of workdays(October 22, 2014) for Case 1, Case 2, Case 3 and Case 4.

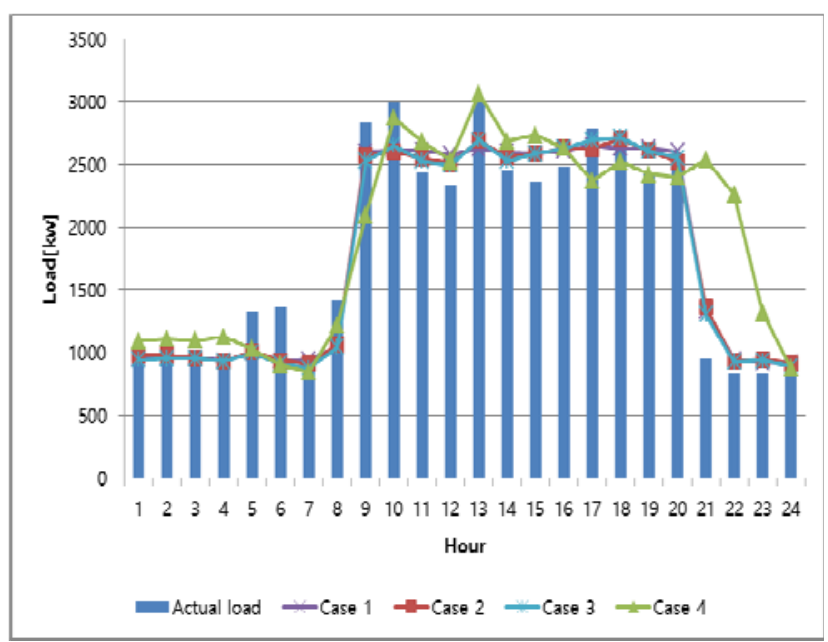

Fig. 3. Load forecasting chart of Workdays

for Case 1, Case 2, Case 3 and Case 4 (October 22, 2014)

\section{B. Load forecasting for the training cases}

Prediction accuracy of neural network varies a lot due to selection of learning time. Through Table 1, learning period is set by 15 days, 30 days and 45 days before the most well predicted case ( 3 input data, 2 neurons in hidden layer).

5) Case 5: number of neuron in hidden layer is 2, number of input data is 3 and learning period is 15 days.

6) Case 6: number of neuron in hidden layer is 2, number of input data is 3 and learning period is 30 days. 
7) Case 7: number of neuron in hidden layer is 2, number of input data is 3 and learning period is 45 days.

Table II. - MAPE of Load forecasting for Case 4, Case 5, Case 6 and Case 7

\begin{tabular}{|c|c|c|c|c|}
\hline & $\begin{array}{c}\text { MAPE of } \\
\text { Case 5 } \\
{[\%]}\end{array}$ & $\begin{array}{c}\text { MAPE of } \\
\text { Case 6 } \\
{[\%]}\end{array}$ & $\begin{array}{c}\text { MAPE of } \\
\text { Case 7 } \\
{[\%]}\end{array}$ & $\begin{array}{c}\text { MAPE of } \\
\text { Case 4 } \\
{[\%]}\end{array}$ \\
\hline $2014-10-01$ & 26.9 & 16.2 & 17.4 & 16.2 \\
\hline $2014-10-02$ & 47.0 & 37.0 & 33.3 & 42.5 \\
\hline $2014-10-06$ & 62.5 & 45.7 & 42.0 & 43.7 \\
\hline $2014-10-07$ & 13.5 & 10.6 & 13.2 & 16.4 \\
\hline $2014-10-08$ & 22.2 & 112.6 & 15.0 & 18.9 \\
\hline $2014-10-10$ & 51.2 & 35.3 & 32.5 & 28.3 \\
\hline $2014-10-13$ & 21.6 & 19.5 & 22.3 & 18.7 \\
\hline $2014-10-14$ & 17.5 & 11.3 & 10.8 & 11.2 \\
\hline $2014-10-15$ & 74.6 & 56.5 & 51.1 & 56.4 \\
\hline $2014-10-16$ & 22.7 & 16.5 & 17.5 & 14.7 \\
\hline $2014-10-17$ & 14.6 & 14.3 & 14.1 & 15.5 \\
\hline $2014-10-20$ & 15.7 & 11.5 & 13.6 & 16.2 \\
\hline $2014-10-21$ & 20.9 & 10.5 & 20.0 & 17.8 \\
\hline $2014-10-22$ & 18.9 & 10.0 & 10.4 & 27.0 \\
\hline $2014-10-23$ & 26.1 & 19.8 & 20.2 & 20.7 \\
\hline $2014-10-24$ & 21.7 & 20.7 & 22.1 & 22.4 \\
\hline $2014-10-27$ & 54.7 & 45.7 & 45.3 & 43.8 \\
\hline $2014-10-28$ & 10.9 & 13.4 & 15.5 & 15.6 \\
\hline $2014-10-29$ & 20.7 & 11.5 & 16.3 & 18.7 \\
\hline $2014-10-30$ & 16.0 & 11.5 & 11.9 & 18.7 \\
\hline Total & 29.0 & 22.2 & 22.2 & 24.0 \\
\hline
\end{tabular}

For the case 6 and case 7 from simulation result, MAPE is the lowest as $22.2 \%$. From Table II, it is possible to confirm that setting before 30 days of predicted date for learning data period is the most accurate to predict load. Fig. 4. show load forecasting chart of workdays(October 22, 2014) for Case 4, Case 5, Case 6 and Case 7.

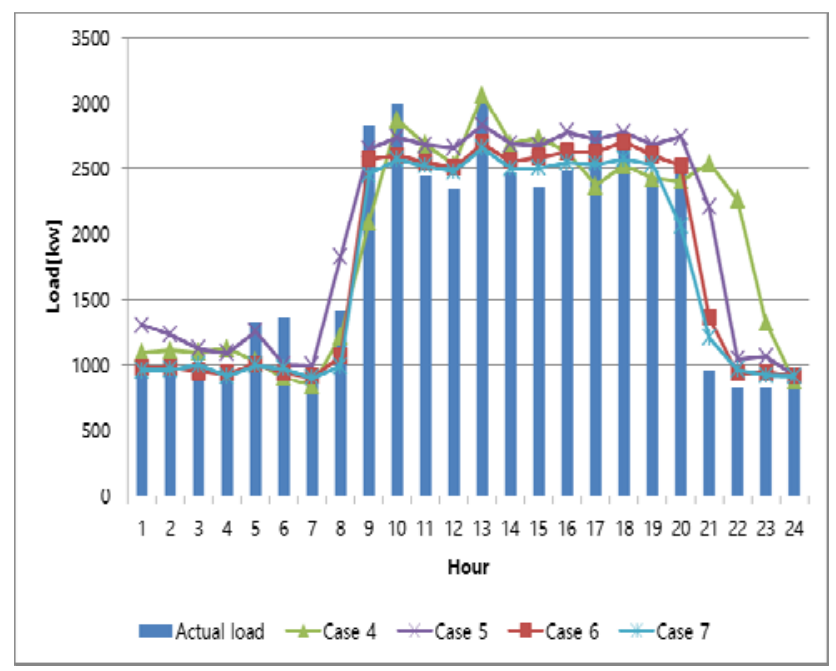

Fig. 4. Load forecasting chart of Workdays for Case 4, Case 5, Case 6 and Case 7 (October 22, 2014)
In this paper, load is predicted to establish optimized charging and discharging plan for demand manageable BESS. After analyze industrial load on October 2014, it is applied to workdays load pattern to conduct experiment. ANN with Feedforward MLP structure is used. It is predicted by applying load into many cases. By using predicted load from ANN and predicted load from average moving method, each error rate of predicted load is calculated and analyzed. For each predicted load, error rate is calculated and compared. It is possible to confirm that prediction error rate varies due to leaning time and number of neuron in hidden layer. Industrial load data that used in this paper as research data existed as very different shape like Figure 2, prediction error rate relatively high, but it is possible to confirm that suggested ANN has the best performance.

For the future research, load pattern of workdays analyzed more to classify patterns and applied to ANN. Then load is predicted and error rate will be calculated. Also it will be applied to both Saturday and holiday load pattern to predict by changing learning time more variously. Also predicted load will be applied to BESS system to confirm efficiency of charging and discharging schedule.

\section{Acknowledgement}

This work was supported by the Power Generation \& Electricity Delivery of the Korea Institute of Energy Technology Evaluation and Planning(KETEP) granted financial resource from the Ministry of Trade, Industry \& Energy, Republic of Korea (No.20143010011830)

\section{References}

[1] D. C. Park, M. A. El-Sharkawi, R. J. Marks, L.E. Atlas and M.J. damborg, "Electric load forecasting using an artificial neural network", Power Systems, IEEE Transactions on, 1991, Vol. 6(2), pp. 442-449.

[2] Ho, Kun-Long, Yuan-Yih Hsu, and Chien-Chuen Yang, "Short term load forecasting using a multilayer neural network with an adaptive learning algorithm", Power Systems, IEEE Transactions on, 1992, Vol. 7(1), pp. 141149.

[3] Hernández, L., Baladrón, C., Aguiar, J. M., Carro, B., Sánchez-Esguevillas, A., \& Lloret, J, "Artificial neural networks for short-term load forecasting in microgrids environment”, Energy, 75, 2014, pp. 252-264.

[4] Henrique Steinherz Hippert, Carlos Eduardo Pedreira, and Reinaldo Castro Souza, "Neural Networks for Short-Term Load Forecasting:A Review and Evaluation" Power Systems, IEEE Transactions on, 2001, Vol. 16(1), pp. 44-55.

[5] Fuller, Robert, "Neural fuzzy systems”, 1995.

[6] Chang-il Kim, In-keun Yu, Y.H, Song, "Kohonen neural network and wavelet transform based apporoach to shortterm load forecasting”, Electric Power Systems Research, 63(3), 2014, pp. 169-176.

[7] Wei, William Wu-Shyong, “Time series analysis”, 1994.

\section{Conclusion}

